\title{
Weak value amplification in resonance fluorescence
}

\author{
U. Satya Sainadh ${ }^{1}$, S. N. Sandhya ${ }^{2}$, Radhika Vathsan ${ }^{3}$ and Andal Narayanan ${ }^{4, *}$ \\ ${ }^{1}$ Center for Quantum Dynamics, Griffith University, Brisbane, QLD 4111, Australia \\ ${ }^{2}$ Miranda House, University Enclave, New Delhi 110007 , India \\ ${ }^{3}$ BITS Pilani (Goa Campus), Zuarinagar, Sancoale, Goa 403 726, India \\ ${ }^{4}$ Light and Matter Physics Group, Raman Research Institute, Bengaluru 560 080, India
}

\begin{abstract}
The concept of weak measurement and associated weak value amplification has sharpened our understanding of the measurement process in quantum mechanics. Recent experiments show that elastic scattering events in resonance fluorescence experiments can exhibit weak value amplification effect, by post-selecting a particular measurement outcome. In this article, we theoretically analyse the physics behind this amplification process. We show that, in general, weak interaction and the associated weak value amplification in resonance fluorescence can be derived from the wellknown theory of spontaneous emission put forth by Wigner and Weiskopff. Using this theory we show that in the elastic scattering regime of resonance fluorescence, weak value amplification helps in segregating rare events. To our knowledge, weak value amplification has not been applied earlier in the time domain as a potential tool to study rare events.
\end{abstract}

Keywords: Quantum mechanics, resonance fluorescence, weak interaction, weak value amplification.

\section{Introduction}

WEAK measurement refers to a measurement process on a quantum system by a measuring device, during which the system experiences very little perturbation. This concept was first introduced by Aharonov et al. ${ }^{1}$. Weak measurement can also be generalized to indicate weak interaction between the system and the observable. It was pointed out subsequently that, under suitable conditions, a projective post-selection of the outcome of interaction can substantially alter the value of the measured observable from its value obtained by measurements without postselection ${ }^{2}$. For nearly orthogonal initial and final states, the weak value of the observable associated with the weak interaction can even get substantially amplified. Such an amplified value for the measured outcome of the observable due to post-selection is referred to as weak value amplification. Weak value amplification was theoretically shown to be useful in situations where the meas-

*For correspondence. (e-mail: andal@rri.res.in) urement is dominanted by technical noise and not by the shot noise of the detector ${ }^{3,4}$.

Experiments demonstrating weak measurements and/or weak interactions have been performed earlier. Using an Young's double slit experimental set-up, the average trajectories of photons exiting either of the two slits, have been mapped ${ }^{5}$. Since weak values can even be complex, it has enabled direct measurement of complex probability amplitudes as is shown in the literature ${ }^{6,7}$. Experiments demonstrating weak value amplification have also been performed. These amplify effects that would otherwise be too small to be measured ${ }^{8,9}$.

In a recent experiment, Shomroni et al. ${ }^{10}$ measured an imaginary weak value in their experiment, which also exhibited weak value amplification for appropriately selected initial and final states of the system. Their system was an atom, initially prepared in a superposition of excited levels of a V-type atomic-level configuration, interacting with its electromagnetic bath environment (Figure 1). After post-selecting the final state of the system by measuring the appropriate polarization state of the elastically scattered light, they showed that for nearly orthogonal initial and final system states, the time of arrival of atoms is delayed compared to the typical timescale of excited state decay.

In this article, we derive mean transit time of atoms when an atom goes from an initial excited state to a designated final state using the well-known Weiskopff-
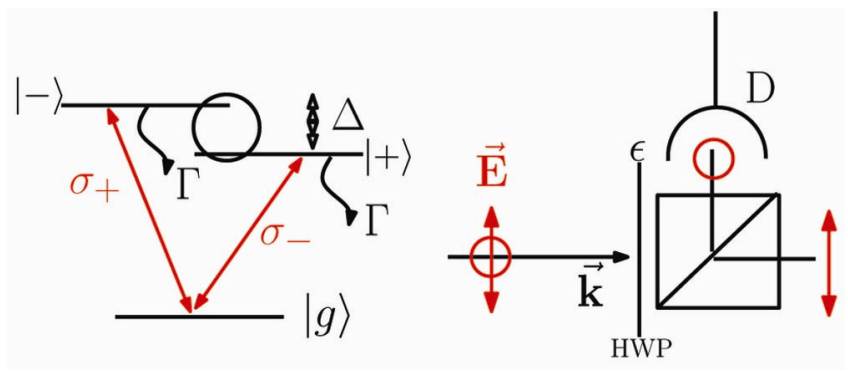

Figure 1. An atomic V-level configuration with ground state $|g\rangle$ and two excited states $|+\rangle$ and $|-\rangle$. The separation between the excited states $\Delta$ is less than $\Gamma$, the rate of excited state decay. The scattered light is detected at $D$, for a particular polarization orientation, selected by suitable adjustment of a half-wave-plate (HWP) at an angle $\varepsilon$. 
Wigner theory of spontaneous emission. Using this theory, we show that the extended excited state lifetime seen in Shomroni et al. ${ }^{10}$ is the time associated with the atom changing from an initial superposition state configuration of excited states to another excited state configuration which is nearly orthogonal to the initial state. Since this is an elastic scattering event, this configuration change occurs without the decay of the atom to the ground state. In regular resonance fluorescence measurements without post-selection, the scattering is dominated by atoms decaying from the excited state to the ground state. We thus show that in this elastic scattering regime of resonance fluorescence, the weak value amplification effect has captured a rare event which would have otherwise not been possible to observe. The simplicity of our theory allows its applicability to systems with multiple (more than two) excited states and allows for experimental observation of excited state configurational changes.

\section{Weak value amplification}

The weak value of an observable is defined as the firstorder truncated value of the observable evaluated between specific initial and final states of the system during a weak interaction ${ }^{11}$.

$$
A_{\mathrm{w}}=\frac{\langle f|\hat{A}| i\rangle}{\langle f \mid i\rangle} .
$$

This value determines the probability of the system going from the initial state $|i\rangle$ to the final state $|f\rangle$ under a unitary evolution (with $\hbar$ taken to be equal to 1 )

$$
\hat{U}(\varepsilon)=\mathrm{e}^{-\mathrm{i} \varepsilon \hat{A}} .
$$

The small parameter $\varepsilon$ makes the interaction of $\hat{A}$ with the system weak. For nearly orthogonal initial and final states and under conditions which still justify the linear truncation of the interaction ${ }^{2}$, the weak value $A_{\mathrm{w}}$ gets amplified. This is called the weak value amplification effect associated with the observable $A$ through a weak interaction defined by the small parameter $\varepsilon$.

\section{Weak value amplification in resonance fluorescence}

Resonance fluorescence typically refers to the scattering of light by an atom when the driving light frequency $\omega$ is close to resonance with the energy level separation $\omega_{0}$ of the atomic levels. There are two main regimes of resonance fluorescence-elastic scattering or Rayleigh scattering regime and inelastic scattering regime ${ }^{12}$. The atomic dipole induced by the incident light can be broken into an average dipole orientation and a fluctuating dipole where the orientation changes randomly (Figure 2). Elastic scattering arises from the non-fluctuating part of the induced dipole when the incident light intensity is low with a saturation parameter $S \ll 1 . S=1$ refers to half the number of atoms being in the excited state at any given point of time.

In the elastic scattering regime, the atom does not change its state of energy. The light is forward-scattered predominantly and the emitted light is phase-coherent with the incident light. In this regime, the phase change undergone by the light is directly correlated to the phase change undergone by the atomic dipole.

In a V-system configuration of the kind shown in Figure 1, a weak scattering regime can be identified when the separation $\Delta$ between excited state levels is taken to be much smaller than $\Gamma$, the line-width of the excited states. This is a weak scattering regime owing to interference effects arising between elastically scattered photons from either of the excited states. Having prepared the initial state of the atom in a particular superposition state of the excited levels ${ }^{10}$

$$
|i\rangle=\frac{|+\rangle+|-\rangle}{\sqrt{2}},
$$

subsequent elastic scattering events can be studied with the atom in the state $|i\rangle$ interacting with the vacuum modes of the bath field surrounding the atom. In elastic scattering regime when the atom does not decay to the ground state, any phase change experienced by the scattered light originates from phase change of this initial superposition state $|i\rangle$. Thus by choosing the appropriate polarization state of the scattered light by a suitable orientation $\theta$ of a polarizer, or a half-wave-plate, we can detect a particular final superposition state $|f\rangle$ of the atom. For weak value amplification effects, this final system state is so chosen that it is nearly orthogonal to the initial state $|i\rangle$.

$$
|f\rangle=\frac{\mathrm{e}^{-\mathrm{i} \varepsilon}|+\rangle-\mathrm{e}^{+\mathrm{i} \varepsilon}|-\rangle}{\sqrt{2}} .
$$

Experimentally, this is achieved by polarization selected measurement of the forward scattered light whose polarization is at an angle $\theta=90^{\circ}+\varepsilon$ to the incident polarization direction at $\theta=0$. Here the value of $\varepsilon$ lies between 0 and 1 .

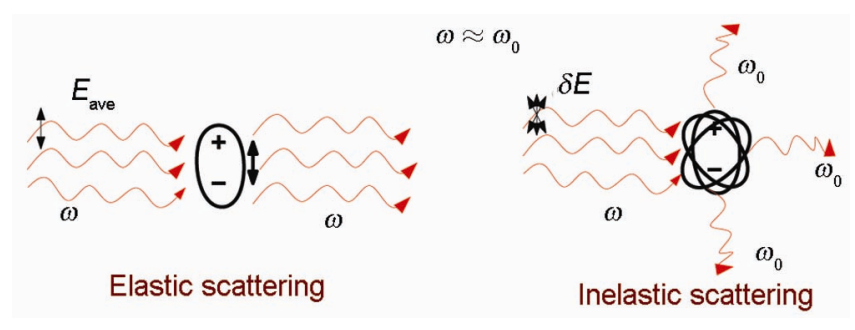

Figure 2. Elastic and inelastic scattering events close to resonance where $\omega \approx \omega_{0}$. 


\section{Weiskopff-Wigner theory for resonance fluorescence from a V-system}

In this section, we give a brief outline of the WeiskopffWigner theory which is used to derive the weak value amplification effect, seen in resonance fluorescence from a V-system ${ }^{10}$. The state of the atom at any time $t$ is given by

$$
\begin{aligned}
|\psi(t)\rangle= & a(t) \mathrm{e}^{-\mathrm{i} w t}\left|\psi_{\text {in, }} 0_{k, s}\right\rangle \\
& +\sum_{\mathbf{k}, s} b_{\mathbf{k}, s}^{1}(t) \mathrm{e}^{-\mathrm{i} w_{k} t}\left|g, 1_{k, s}\right\rangle \\
& +\sum_{\mathbf{k}^{\prime}, s^{\prime}} b_{\mathbf{k}^{\prime}, s^{\prime}}^{2}(t) \mathrm{e}^{\left.-\mathrm{i} w_{k^{\prime}}, t\right)}\left|g, 1_{k^{\prime}, s^{\prime}}\right\rangle .
\end{aligned}
$$

Here $a(t)$ refers to the probability amplitude that the atom is in the excited state configuration $\left|\psi_{\text {in }}\right\rangle$ with no photons. The terms $b_{k, s}^{1,2}$, refer to the probability amplitudes that the atom decays to the ground state $|g\rangle$ from either of the excited states, emitting a photon in the appropriate mode labelled by the propagation vector of the emitted photon $\mathbf{k}$ or $\mathbf{k}^{\prime}$ and its polarization state $s$ or $s^{\prime}$. We take the atom to be initially in the state $|i\rangle$ given by eq. (3). Thus $\left|\psi_{\text {in }}\right\rangle=|i\rangle$. The atom is surrounded by the vacuum modes of the electromagnetic bath field. The Hamiltonian of this system of atom and bath is given by

$$
\begin{aligned}
H / \hbar= & w_{+} \sigma_{++}+w_{-} \sigma_{--}+\sum_{k, s} w_{k} \hat{n}_{k, s} \\
& -\sum_{k, s} g_{k, s}^{1} \sigma_{+g} \hat{a}_{k, s}-\sum_{k^{\prime}, s^{\prime}} g_{k^{\prime}, s^{\prime}}^{2} \sigma_{-g} \hat{a}_{k^{\prime}, s^{\prime}}+\text { c.c., }
\end{aligned}
$$

where $\sigma_{i j}=|i\rangle\langle j|$ and

$$
g_{k, s}=-i \sqrt{\frac{w_{k}}{2 \hbar \varepsilon_{0} V}}\left(\mathbf{d} \cdot \varepsilon_{\mathbf{k}, \mathbf{s}}\right),
$$

is the coupling of the electromagnetic modes of the bath with the excited state of the atom. Here $\mathbf{d}$ is the induced dipole moment vector and $\varepsilon_{\mathbf{k}, \mathbf{s}}$ is the unit vector representing the polarization direction. $w_{+}$and $w_{-}$refer to the energies of the excited states $|+\rangle$ and $|-\rangle$, with ground state energy taken to be the zero of the energy scale. Consider the Schrödinger wave equation

$$
H|\psi(t)\rangle=i \hbar \frac{\partial|\psi\rangle}{\partial t}
$$

In order to evaluate the probability that the atom which was in $|i\rangle$ state at $t=0$, gets elastically scattered at a later time to the nearly orthogonal $|f\rangle$ state, we take the inner product of eq. (6) with $\left\langle f, 0_{k, s}\right|,\left\langle g, 1_{k, s}\right|$ and $\left\langle g, 1_{k^{\prime}, s^{\prime}}\right|$ separately on both sides. This gives us

$$
\begin{aligned}
& \dot{a}(t)=-i\left(\frac{w_{+}+w_{-}}{2}-w\right) a(t)+\frac{\Delta \cot \varepsilon}{2} a(t) \\
& +\frac{i}{\sqrt{2}} \sum_{k, s}\left(b_{k, s}^{1}(t)+b_{k, s}^{2}(t)\right)\left(g_{k, s}^{1}-g_{k, s}^{2}\right) \mathrm{e}^{-\mathrm{i}\left(w_{k}-w\right) t}, \\
& \dot{b}_{k, s(t)}^{1}=\frac{i a(t)}{\sqrt{2}}\left(g_{k, s}^{1 *}-g_{k, s}^{2 *}\right) \mathrm{e}^{\mathrm{i}\left(w_{k}-w\right) t}-i w_{k} b_{k, s}^{2}(t), \\
& \dot{b}_{k^{\prime}, s^{\prime}}^{2}(t)=\frac{i a(t)}{\sqrt{2}}\left(g_{k^{\prime}, s^{\prime}}^{1 *}-g_{k^{\prime}, s^{\prime}}^{2 *}\right) \mathrm{e}^{\mathrm{i}\left(w_{k^{\prime}}-w\right) t}-i w_{k^{\prime}} b_{k^{\prime}, s^{\prime}}^{1}(t) .
\end{aligned}
$$

By defining $b_{k, s}^{1}(t)+b_{k, s(t)}^{2}=b_{k, s}(t)$ and $g_{k, s}^{1}-g_{k, s}^{2}=$ $g_{k, s}$ and using the fact that the separation between the excited states is less than that of the width of their energy level, i.e. $\Delta \ll \Gamma$, we take $w_{+} \approx w_{-}$, thereby making $w=\left(w_{+}+w_{-}\right) / 2$. This reduces the equations to a simpler form given by

$$
\begin{aligned}
& \dot{a}(t)=\frac{i}{\sqrt{2}} \sum_{k, s} b_{k, s}(t) g_{k, s} \mathrm{e}^{-\mathrm{i}\left(w_{k}-w\right) t}+\frac{\Delta \cot \varepsilon}{2} a(t), \\
& \dot{b}_{k, s}(t)=\sqrt{2} i a(t) g_{k, s}^{*} \mathrm{e}^{\mathrm{i}\left(w_{k}-w\right) t}-i w_{k} b_{k, s}(t) .
\end{aligned}
$$

We solve this coupled set of equations by taking the summation over two orthogonal circular polarization states which give rise to the corresponding dipole moments and over the continuum of $k$ states. Since $\varepsilon$ is small, we replace $\cot \varepsilon$ by $1 / \varepsilon$. We then obtain for $a(t)$

$$
\begin{aligned}
& \dot{a}(t)=-\frac{\Gamma}{2} a(t)+\left(\frac{\Delta}{2 \varepsilon}\right) a(t), \\
& a(t)=a(0) \exp \left(-\Gamma t / 2\left(1-\frac{\Delta}{\Gamma \varepsilon}\right)\right),
\end{aligned}
$$

where $\Gamma=\left(\omega^{3}|\alpha|^{2}\right) /\left(3 \pi \varepsilon_{0} c^{3} \hbar\right)$ and $a(0)=1$. Here $a(t)$ represents the probability amplitude that the atom which was in state $|i\rangle$ will get elastically scattered to state $|f\rangle$. Plotting the modified lifetime

$$
\tau=\frac{1}{\Gamma\left(1-\frac{\Delta}{\Gamma \varepsilon}\right)}
$$

with experimental values of $\Delta=600 \mathrm{kHz}$ and $\Gamma=6 \mathrm{MHz}$ reported in Shomroni et al. ${ }^{10}$, we get the mean lifetime of atoms going from $|i\rangle$ to $|f\rangle$ as a function of $\varepsilon$ as shown in Figure 3. It is clearly seen from the plot that for small 


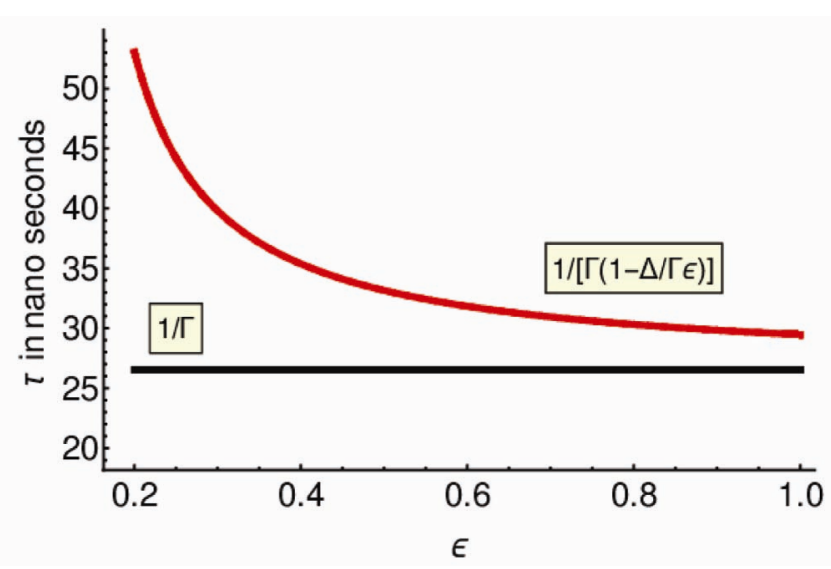

Figure 3. The red line plot shows increased mean lifetime $\tau$ of atoms as they scatter from an initial state $|i\rangle$ to a final state $|f\rangle$, as a function of $\varepsilon$. Different values of $\varepsilon$ denote different final states $|f\rangle$. The black line shows the decay timescale of the atom to go from $|i\rangle$ to the ground state $|g\rangle$ in the absence of post-selection.

values of $\varepsilon$, the mean lifetime increases compared with the decay lifetime $1 / \Gamma$ during which the atom decays from the excited state to the ground state. This effect of increase in mean lifetime was measured and reported in figure $3 b$ of Shomroni et al. ${ }^{10}$ and a remarkable qualitative and quantitative agreement between our theory and the experimental result is seen.

Using our theory, we see that, as the $|f\rangle$ state becomes more and more orthogonal to the initial state $|i\rangle$, the time taken for scattering between the states increases. Without post-selection, these events would not have been possible to observe as the scattering will be dominated by events with a typical timescale ${ }^{13}$ of $1 / \Gamma$ that takes the atom from $|i\rangle$ to $|g\rangle$. Thus we have found that by appropriate choice of post-selected states in a weak interaction regime, it is possible to single out a rare event using weak value amplification effect.

\section{Conclusion}

Under resonant interactions between atom and light, elastic scattering events dominate in the low saturation limit. These scattering events coherently map phase changes experienced by induced atomic dipoles to the phase of the scattered light. A weak interaction regime in elastic scattering of light from a three-level V-type atomic configuration of levels is established, when the separation between the two closely spaced excited states is very small compared to the decay rate of excited state atoms to the ground state. We show using the Weiskopff-Wigner theory of spontaneous emission, that in such a weak scattering regime an increase in lifetime occurs for events where an observable takes the atom between nearly orthogonal excited state superpositions. The increased time signifies the rarity of such scattering events. Thus our theory brings out the fact that, it is possible to single out a rare event using weak value amplification which would otherwise not be possible to observe. We have thus shown that weak value amplification can be applied in the time domain. This opens the possibility to observe the otherwise impossible-to-observe excited state configurational changes before the atom decays to the ground state.

1. Aharonov, Y., Albert, D. Z. and Vaidman, L., How the result of a measurement of a component of the spin of a spin-1/2 particle can turn out to be 100. Phys. Rev. Lett., 1988, 60(14), 1351-1354.

2. Duck, I. M., Stevenson, P. M. and Sudarshan, E. C. G., The sense in which a 'weak measurement' of a spin-1/2 particle's spin component yields a value 100. Phys. Rev. D, 1989, 40(6), 21122117.

3. Brunner, N. and Simon, C., Measuring small longitudinal phase shifts: weak measurements or standard interferometry? Phys. Rev. Lett., 2010, 105(1), 010405-1 to 010405-4.

4. Feizpour, A., Xing, X. and Steinberg, A. M., Amplifying singlephoton nonlinearity using weak measurements. Phys. Rev. Lett., 2011, 107(13), 133603-1 to 133603-5.

5. Kocsis, S., Braverman, B., Ravets, S., Stevens, M., Mirin, R. P., Shalm, L. K. and Steinberg, A. M., Observing the average trajectories of single photons in a two-slit interferometer. Science, 2011, 332(6034), 1170-1173.

6. Lundeen, J. S., Sutherland, B., Patel, A., Stewart, C. and Bamber, C., Direct measurement of the quantum wavefunction. Nature, 2011, 474(7350), 188-191.

7. Salvail, J. Z., Agnew, M., Johnson, A. S., Bolduc, E., Leach, J. and Boyd, R. W., Full characterization of polarization states of light via direct measurement. Nature Photon., 2013, 7(4), 316321.

8. Hosten, O. and Kwiat, P., Observation of the spin Hall effect of light via weak measurements. Science, 2008, 319(5864), 787-790.

9. Dixon, P. B., Starling, D. J., Jordan, A. N. and Howell, J. C., Ultrasensitive beam deflection measurement via interferometric weak value amplification. Phys. Rev. Lett., 2009, 102(17), 173601-1 to 173601-4.

10. Shomroni, I., Bechler, O., Rosenblum, S. and Dayan, B., Demonstration of weak measurement based on atomic spontaneous emission. Phys. Rev. Lett., 2013, 111(2), 023604-1 to 023604-5.

11. Dressel, J., Malik, M., Miatto, F. M., Jordan, A. N. and Boyd, R. W., Understanding quantum weak values: basics and applications. Rev. Mod. Phys., 2014, 86(1), 307-316.

12. Mollow, B. R., Power spectrum of light scattered by two-level systems. Phys. Rev., 1969, 188(5), 1969-1975.

13. Zurek, W. H., Decoherence and the transition from quantum to classical - Revisited. arXiv:quant-ph/0306072.

doi: $10.18520 /$ v109/i11/2002-2005 